\title{
Computer assisted analysis of echocardiographic image sequences
}

\author{
Andrea Giachetti ${ }^{1}$, Marco Cappello ${ }^{2}$, Guido Gigli ${ }^{3}$ and Vincent Torre ${ }^{1}$ \\ 1 Dipartimento di Fisica Università di Genova. \\ 2 Consorzio I.N.F.M. - Universita' di Genova \\ Via Dodecaneso 33, 16146 Genova \\ Tel: 39-10-3536311 Fax: 39-10-314218 \\ ${ }^{3}$ Servizio di Cardiologia, Ospedale di Rapallo, Piazza Molfino 10, 16035 Rapallo(GE) \\ Tel: 39-185-683231 Fax: 39-185-683277 \\ E-mail: giachetti@genova.infn.it
}

\begin{abstract}
In this paper we present a semi-automatic system for the analysis of echocardiographic image sequences, able to provide useful information to cardiologists. The proposed approach combines well known techniques for the detection of left ventricular boundaries with the computation of optical flow. The initial detection of the cavity contour is based on an improved balloon model, the computation of optical flow is performed with a correlation technique and the contour tracking is obtained combining motion information provided by the optical flow with a snake-based regularization. The system is able to follow precisely the cavities motion, to provide several quantitative features of the heart beat and a dynamic representation of systolic and diastolic motion. Preliminary experimental results are presented and commented with particular attention to their clinical relevance.
\end{abstract}

\section{Introduction}

Echocardiography is the commonest technique for cardiac imaging and provides a large quantity of clinical information on cardiac structures and functions in an incruent manner. The evaluation of the left ventricle is one of the most valuable clinical application of echocardiography [1]; its volume and its ejection fraction have an extraordinary diagnostic and prognostic value [2]. In ischemic heart disease, echocardiography is useful in the diagnosis of myocardial infarction and transient ischemic episodes [3]. The analysis of echocardiographic image sequences is usually performed by visual inspection. However, some systems are able to produce image processing helpful to enhance the definition of the heart cavities and to provide some other useful parameters [4]. Computer Vision tools have been used in pilot studies to perform heart cavity detection: interpolations of calculated edges $[5,9]$, snakes $[7,9]$ and segmentation techniques based on Markov Random Fields [10] have already been exploited, while optical flow has been used to study heart motion $[12,13]$. In this paper we describe some preliminary results of a system for the automatic analysis of echocardiographic images, based on the use of snakes [6,7] and the computation of optical flow [11]. This system detects the left ventricular contour on final echocardiographic images (i.e. without any intervention in the processing cascade of ultrasonic signal), computes its area, tracks it and analyses its motion. 


\section{The detection of ventricular cavities}

The analysis of the image sequence starts with the detection of the left ventricular cavity in the first image. This function is performed by an active contour model, inspired by the recent work on snakes [6] and balloons [7]. The contour is represented by a closed curve, parametrized by the arc length $s: \mathbf{p}(s)=(x(s), y(s))$. In our model, similar to the balloon model of Cohen \& Cohen [7], the initial shape of the contour can be initialized by hand near the true boundaries or just giving the center of the cavity (see Section 4); in this case the initial contour is taken as a small ellipse centered in the given point. The curve undergoes an evolution driven by an internal force $\mathbf{F}_{i n t}=\alpha \mathbf{p s s}_{s}$ tending to minimize the contour length, an inflating force $\mathbf{F}_{g}=f_{g} \gamma$ (where $\gamma(s)$ is the unit normal vector of the curve) tending to enlarge it, and a deflating force that stops the contour expansion near the cardiac boundaries. This force has been introduced because, due to noisiness and low contrast of echocardiographic images, the use of the usual edge force $\mathbf{F}_{\text {edge }}(\mathbf{p})=-\nabla|\nabla E(\mathbf{p})|$. (where $E(\mathbf{p})$ is the grey level at the point $\mathbf{p}$ ) was not convenient. The new force is defined as follows:

$$
\mathbf{F}_{d}=-f_{d}\left(1-\left(\exp \left(\frac{E(\mathbf{p})-k}{T}\right)-1\right)^{-1}\right) \boldsymbol{\gamma}
$$

This is a smooth step function where $T$ controls the smoothing, $k$ is the center of the small region where its value changes from zero to $f_{d}$. When the grey level is low (inside the cavity), the deflating force is approximately zero. When the grey level is above the threshold $k$, the force quickly reaches the maximum value $f_{d}$ and if the bright region is sufficiently large, the contour is stopped at its border.

The implementation of the active contour is made substituting the curve $\mathbf{p}(s)$ with a closed chain $\mathbf{p}(i)$ of $\mathrm{N}$ points, replacing the derivatives of the internal force with finite differences and approximating the other forces with vectors applied to the points. Finally the points are simultaneously shifted of the quantity obtained giving unitary masses to the points and computing their approximated dynamics for a unitary time step. The procedure is then iterated until the growth of the contour is stopped.

\section{Cardiac walls motion and contour tracking}

The analysis of the motion of the ventricular contour can be useful to build a model of the complex motion of the heart and to detect the presence of ischemic regions from irregular motions during the contraction. Given the chain representing the initial ventricular boundaries, we simply compute the optical flow at each point of the chain. The procedure used for our system is the following: after a simple smoothing of the images with a gaussian filter $\exp \left(-\left(x^{2}+y^{2}\right) / 2 \sigma^{2}\right)$ with a value of 1.5 pixels for $\sigma$, the point $\left(x^{\prime}, y^{\prime}\right)$ at time $t+1$ corresponding to point $(x, y)$ at time $t$ is the one with the lowest value of the quantity:

$$
\sum_{i=-n}^{n} \sum_{j=-n}^{n}\left(E\left(i+x^{\prime}, j+y^{\prime}, t+1\right)-E(i+x, j+y, t)\right)^{2}
$$

where $E(x, y, t)$ is the grey level of pixel $(x, y)$ of the frame labelled by the integer variable $t$. Usually the value of $n$ was set equal to 15 . The displacements 
computed at the chain points can be used to compute the new contour for the next image, by simply shifting the points according to their values. This procedure, however, is not accurate enough to produce a reliable detection of the new ventricular contour: the existence of erroneous displacements and the presence of the quantization noise can generate some problems. If the wrong matches are not eliminated, after a few frames the contour becomes irregular, with many points far from the cardiac walls. To overcome this problem, after shifting the contour points with the computed displacements, we applied a regularization procedure consisting in a few iterations of the forces described in Section 1. In this way, there is a fast convergence towards the true cardiac walls. To avoid that during the regularization even the points where the computed optical flow is correct undergo large displacements, the masses of the point are no longer unitary, but are taken inversely proportional to an appropriate confidence measure of the flow.

\subsection{Area evolution and ejection fraction}

A useful parameter for the analysis of heart functionality is the area $S$ of the computed ventricular section. For each computed contour $p(i)$, an application of Gauss-Green's theorem gives the following formula for the area:

$$
S=\frac{1}{2} \sum_{1}^{N} \mathbf{p}(i) \cdot \gamma(i)|\mathbf{p}(i)-\mathbf{p}(i-1)|
$$

The area of the cavity section can be used also to estimate ventricular volumes and important functional parameters such as the ejection fraction [2].

\section{Experimental results}

The proposed approach was tested on several image sequences of healthy and anomalously beating hearts acquired from a video used for cardiologists training. The aim of this experimentation was to establish whether the proposed approach was able to track the left ventricle reliably and to evaluate whether the description of cavity motion with the optical flow was useful. The performance of the system depended rather critically, as it might be expected, on the numerical values of the different parameters used. Contours chains were usually formed by 100 points. In order to have a strong but short range deflating force and an equilibrium position near the true boundaries of the cavity the values of the parameters $T$ and $f_{r}$ were both set equal to 1 . The range of the inflating and internal forces was rather short, approximately of the order of 0.1 pixels and their ratio was tuned for each sequence to have the better results. The choice of the threshold $k$ was very important and had to be differently made for each sequence; furthermore $k$ should not be constant for all the points, because in the echo images the gray level corresponding to the cardiac membrane depends on the angle of incidence of the ultrasound and on the gain factors. This means that the gray value corresponding to the membrane is different for different contour points and can change with time. This do not affect too much the optical flow algorithm, but can create more problems for the image force driving the snake. To overcome this problem we introduced local values of the threshold $k$. If the curve was initialized by hand, the thresholds were automatically chosen with 

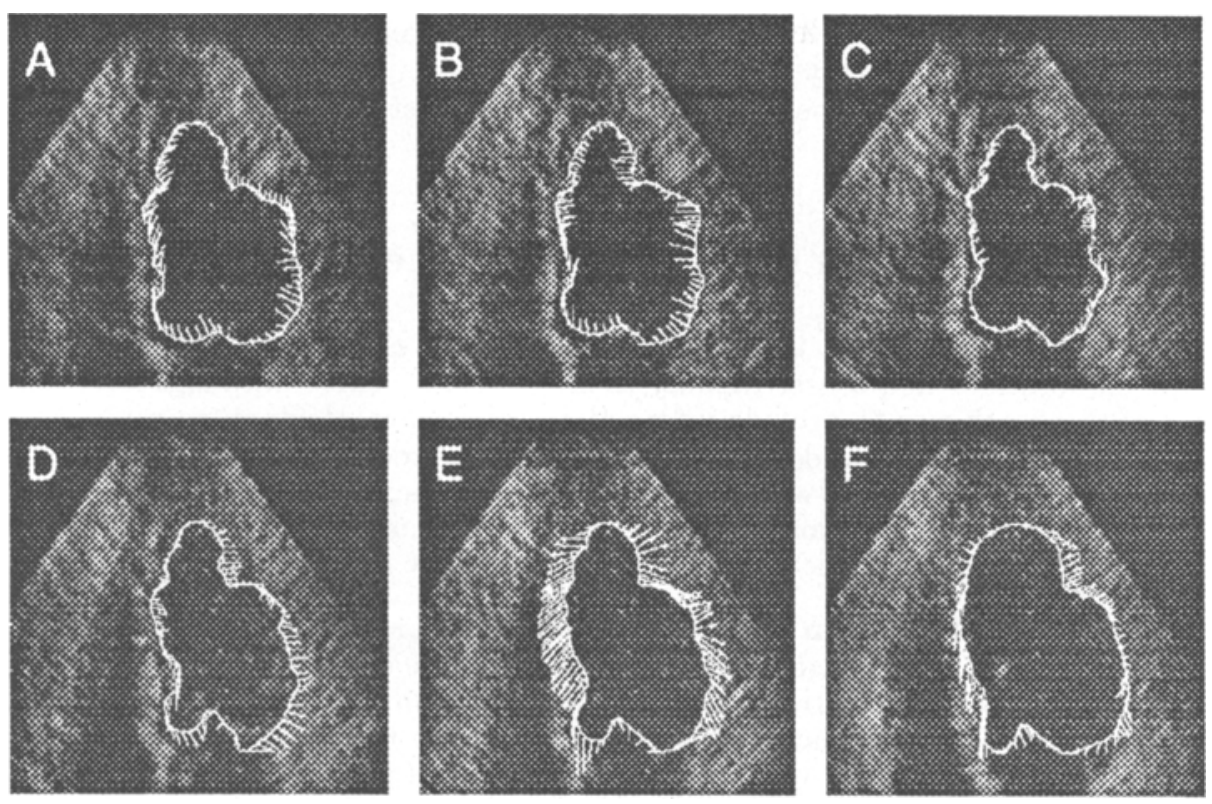

Fig. 1. A, B, C: Contour detection, tracking and optical flow computation for three consecutive frames of systolic motion of the left ventricle. D,E,F: The same for diastolic motion. Flow vectors are magnificated by a factor 2 .

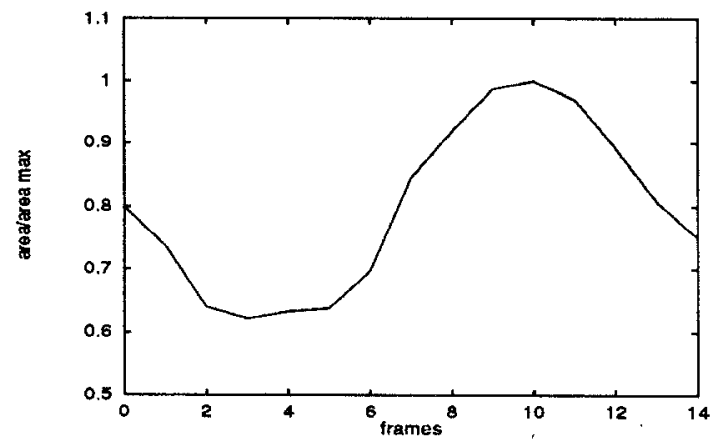

Fig. 2. A: The evolution of the area during the whole heart beat of Fig.1.

values close to the average gray level of the image outside the curve near each point. This adaptive choice was then repeated at each frame, so that the system was able to tolerate changes in the brightness of the cardiac boundaries. If the contour was initializated giving the center of the cavity, we left the possibility to choose four different thresholds for the upper, the left, the right and the lower part of the contour for the first contour detection.

The computation of optical flow of points lying on the cavity walls provided correct vector displacements for most of the points. Displacements were rather large because of the low acquisition rate (25 frame/s.). Fig. 1 shows the initial contour detection (A) and during a whole beat, where the computed boundaries of the ventricular cavity are superimposed to the corresponding images together with the optical flow vectors. The tracking of the contour was obtained shifting 
the chain points with the described technique. After an entire beat (in this case 14 frames), the contour was approximately in the original position. 2).

The computed area of the cross section had a correct periodic behaviour (Fig.

\section{Interaction with medical doctors: the graphic tool}

Once we had a way to track the contour and compute its motion we developed some elaborations and a user-friendly tool for an interactive data analysis. The aim of this work was to provide information for medical doctors, so we had to consider cardiologists' needs to build an interactive tool able to realize an easy initialization of the model with a correct tuning of the parameters, and to show as final results all the elaborations of the computed contours and of the motion vectors that can help medical diagnosis. These are the main features of the tool:

- Contour initialization and tuning of the parameters The user can either choose the initialization by hand, starting the contour by clicking with the mouse near the ventricle contour or the central initialization, just clicking with the mouse inside the ventricle and then tuning the parameters (number of contour points, force values ...) with the cursors on a dedicated panel to reach the correct result. The user can also choose different parameters for the contour following, turn on and off the optical flow computation, change the mask size and the search space for the correlation and so on. When the analysis is started, the graphic display shows the evolution of the contour and the flow vectors superimposed to the correspondent frames.

- Contour envelope display The envelope of the contour during a beat is traced during the evolution to show the entity of the motion of the ventricular boundaries with a colour code indicating the absolute value of the speed. When a region of the border is moving fast, the contour is traced with a bright colour (yellow, red), while when its motion is slow, the contour is traced with a dark colour. If a region of the contour where the quality of the image is sufficiently good is an ischemic one, this plot can evidentiate it (Fig. 3C).

- Area/Volume display The computed area of the ventricular cross-section is plotted in a dedicated window. The value of the area can be replaced by the volume estimated with an appropriate approximation. Values can also represent the true dimension of the cavity by setting the pixel $/ \mathrm{cm}$. ratio clicking on the reference points in the first image. The estimated ejection fraction is also given at the end of the analysis.

- Anomalous motion Regions where the motion of the contour is not regular are detected by studying the correlation between the computed evolution of the area and the motion of the contour points. Different values of this measure are represented with different colours on the representation of the contour envelope. If the computed flow is sufficiently reliable, this graphic can be used to detect diskinesia (Fig. 3D). 


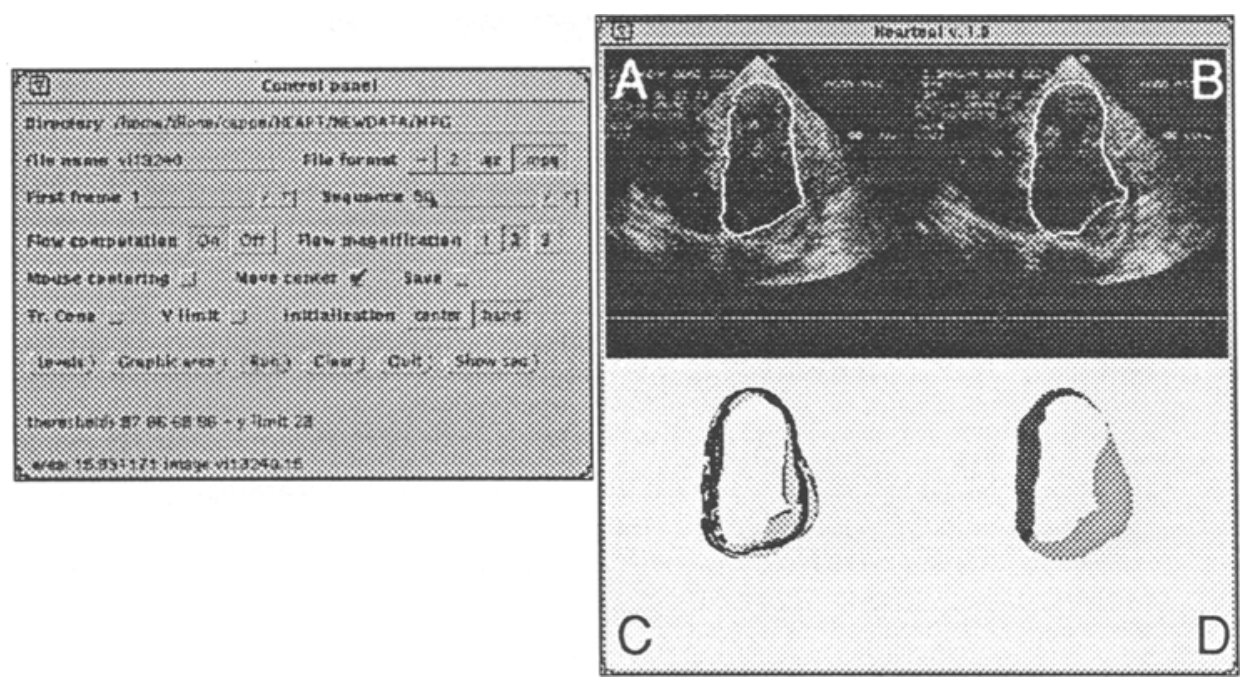

Fig. 3. The main panel and the graphic display of the tool used for the interactive analysis of ventricular boundaries motion. A shows the optical flows relative to the maxima of the expansion and the contraction; $C$ represent the contour envelope with trace of speed (darker colour = slower motion), while in D the darker region represents the detection of anomalous motion. In this case, a slow and anomalous motion is detected on the upper left region, result comfirmed by the analysis of a cardiologist.

\section{Discussion}

The proposed approach for the analysis of echocardiographic image sequences is based on optical flow, that is used for three reasons: to improve the accuracy of the tracking of the cavity walls, to show the complex sequence of ventricular contractions, and as an independent test for contraction abnormalities, as shown in Fig. 3. Indeed, the computation of optical flow restricted on the points on the cavity walls, provides a good description of the dynamics of the heart and therefore of its functional state. In previous studies the computation of the optical flow on echocardiographic image sequences was impaired by several problems because of noise [12]. The computation of the flow over the entire image requires also a long computing time, which is not suitable for real-time clinical application. We solved these problems by computing motion only in the contour points and by using a robust correlation-based technique able to provide good results even in the presence of noise and even in the case of large shifts of the contour in successive frames. The preliminary results obtained with our system are promising: with good quality images the system identified wall motion abnormalities and the left ventricle area changes occurring through the cardiac cycle. The method is also sufficiently fast for clinical applications: the computation of flow vectors and the regularization of the contour are performed in about one second for each frame on a Sun 10 workstation. 


\section{References}

1. S.J. Mason and N.J. Fortuin, "The use of echocardiography for quantitative evaluation of left ventricle function" Prog. Cardiovasc. Dis. 21: 119 (1978)

2. M.A. Quinones et al. "A new simplified method for determining ejection fraction with two-dimensional echocardiography" Circulation 64:744 (1981).

3. R. E. Kreber, M. L. Marcus, "Evaluation of regional miocardial function in ischemic heart disease by echocardiography". Prog. Cardiovasc. Dis. 20:41 (1978).

4. V. F. Vanderberg et al. "Estimation of left ventricular cavity area with an on line, semiantomated echocardiographic edge detection system" Circulation 86:159 (1992).

5. C. H. Chu, E.J. Delp and A.J. Buda, "Detecting Left Ventricular Endocardial and Epicardial Boundaries by Digital Two-Dimensional Echocardiography" IEEE Trans. on Medical Imaging 7:2 266 (1988)

6. A. Kass, A. Witkin and D. Terzopoulos, "Snakes: Active contour models," Int. J. of Comp. Vision 1, 321-331 (1988).

7. L.D. Cohen and Isaac Cohen, "A finite element method applied to new active contour models and $3 \mathrm{D}$ reconstructions from cross-sections" Proc. of 3rd Int. Conf. on Comp. Vision, pp. 587-591 (1990).

8. A. Blake et al. "Affine-invariant contour tracking with automatic control of spatiotemporal scale" Proc. 4th I.C.C.V. pp. 66-75 (1993).

9. I. L. Herlin and N. Ayache, "Features Extraction and Analysis Methods for sequences of Ultrasound Images" Proc. 2nd E.C.C.V., pp. 43-55 (1992).

10. I. L. Herlin, D. Breziat, G. Giraudin, C. Nguyen and C. Graffigne, "Segmentation of echocardiographic images with Markov random fields" Proc. 3rd E.C.C.V., 2 pp. 201-206 (1994).

11. B.K.P. Horn and B.G. Schunck, "Determining optical flow," Artificial Intelligence 17, 185-203 (1981).

12. G. E. Mailloux, A. Bleau et al., "Computer Analysis of Heart Motion from TwoDimensional Echocardiograms" IEEE Trans. Biom. Eng. 34:5 356 (1987).

13. S. C. Armatur and H. J. Vesselle, "A New Approach to Study Cardiac Motion: The Optical Flow of Cine MR Images". Nuclear M. R. in Medicine 12, 59-67. 